\title{
CDK6 and miR-320c Co-Regulate Chondrocyte Catabolism Through NF-kB Signaling Pathways
}

\author{
Hao Sun Zhiyu Huang Peihui Wu Zongkun Chang Weiming Liao \\ Zhiqi Zhang \\ Department of Joint Surgery, The First Affiliated Hospital of Sun Yat-sen University, Guangzhou, China
}

\section{Key Words}

$\mathrm{Cdk} 6 \cdot \mathrm{miR}-320 \mathrm{c} \cdot$ Chondrogenesis $\cdot$ Chondrocytes inflammation

\begin{abstract}
Background/Aims: Cyclin-dependent kinase 6 (CDK6) regulates inflammatory response and cell differentiation. This study sought to determine whether CDK6 and miR-320c co-regulate chondrogenesis and inflammation. Methods: Utilizing quantitative real-time PCR (qRT-PCR) and immunohistochemistry (IHC), CDK6 and miR-320c expression were assessed in a micromass culture of human bone mesenchymal stem cells that underwent chondrogenesis in vitro as well as in chondrocytes from E16.5 mouse forelimbs. Normal chondrocytes were transfected with miR-320c mimic, miR-320c inhibitor, or CDK6-siRNA. Luciferase reporter assay results confirmed that miR-320c directly targets $C D K 6$ by interacting with the $3^{\prime}$-untranslated region (3'-UTR) of its mRNA. qRT-PCR, Western blotting, and Cell Counting Kit-8 were subsequently used to evaluate the effects of miR-320c overexpression and CDK6 inhibition on inflammatory factor expression, as well as to investigate the effects of NF-kB and MAPK signaling pathway activation on IL-1 $\beta$-induced chondrocyte inflammation. Results: Our results show that miR320c expression increased during the middle stage and decreased during the late stage of hBMSC chondrogenic differentiation. In contrast, CDK6 expression decreased during the middle stage and increased during the late stage of hBMSC chondrogenic differentiation. Moreover, CDK6 expression increased in severe OA cartilage and in hypertrophic chondrocytes of mouse forelimbs at E16.5. Results of the luciferase reporter assay showed that miR-320c modulated CDK6 expression by binding to the 3'-UTR of its mRNA. miR-320c overexpression and CDK6 inhibition repressed IL-1 $\beta$-induced expression of inflammatory factors and regulated the NF-kB signaling pathway. Conclusion: CDK6 and miR-320c co-regulate hBMSC chondrogenesis and IL-1 $\beta$-induced chondrocyte inflammation through the NF-kB signaling pathway, suggesting that miR-320c and CDK6 inhibitors can be used to repress catabolism in human chondrocytes.

H. Sun and Z. Huang contributed equally to this work.

Weiming Liao

and Zhiqi Zhang
Dept. of Orthopaedic Surgery, The $1^{\text {st }}$ Affiliated Hospital of Sun Yat-sen University

\#58 Zhongshan 2nd Road, Guangzhou 510080 (China)

E-Mail liaowmsysu@163.com; zhzhiqi@mail.sysu.edu.cn 


\section{Cellular Physiology Cell Physiol Biochem 2018;51:909-923 and Biochemistry \begin{tabular}{l|l} 
DOI: 10.1159/000495392 & $\begin{array}{l}\text { (c) } 2018 \text { The Author(s). Published by S. Karger AG, Basel } \\
\text { www.karger.com/cpb }\end{array}$
\end{tabular}

\section{Introduction}

Proinflammatory cytokines derived from the synovium play a crucial role in osteoarthritis (OA) development [1, 2]. Interleukin-1 $\beta$ (IL-1 $\beta$ ), one of the most common proinflammatory cytokines, disrupts the homeostasis between anabolism and catabolism both by upregulating matrix metalloproteinase (MMP), IL-6, and IL-8 expression and nitric oxide (NO) synthesis and by downregulating proteoglycan and collagen synthesis [3, 4]. IL$1 \beta$ levels are significantly increased in chondrocytes and synoviocytes of patients with $0 A[5$, 6], indicating its importance as a therapeutic target.

Cyclin-dependent kinase 6 (CDK6) and its homologue CDK4 belong to the CDK family, which includes proteins that regulate the cell cycle and promote the G1/S transition. Previous studies have reported that CDK6 inhibition represses cancer progression, especially in breast cancer patients $[7,8]$. However, results of a series of studies have challenged this classical role of CDK4 and CDK6 in cell cycle regulation due to the findings that individual or combined deletion of $C D K 4$ and $C D K 6$ do not affect cell proliferation $[9,10]$. Furthermore, findings that CDK6 is activated independently of CDK4 by an unknown upstream kinase and that aberrant CDK6 expression or activation contributes to chronic inflammation through the NF-kB signaling pathway suggest that CDK6 performs other roles in addition to regulating the cell cycle $[11,12]$. To this end, previous studies have shown that some CDK family proteins play a role in proinflammatory cytokine-induced inflammation. For example, proinflammatory cytokines such as IL-1 $\beta$ and TNF- $\alpha$ activate CDK9, and CDK9 inhibition protects human cartilage cells from catabolic effects without affecting their viability and function [13].

MicroRNAs (miRNAs or miRs) are short ( $22 \mathrm{nt}$ ), non-coding, single-stranded RNAs that function as post-transcriptional regulators. miRNAs silence gene expression by binding to the $3^{\prime}$-untranslated region (3'-UTR) of target mRNAs, thereby promoting their degradation or inhibiting their translation [14]. Previously, we identified miRNAs that were highly expressed during the chondrogenic differentiation of human adipose-derived stem cells [15]. In addition, we found that these miRNAs regulated cartilage inflammation, development, and degeneration [16].

Recent studies have shown that miR-320c is an important regulator of inflammation [17-19]. We previously found that miR-320 regulates IL-1 $\beta$-induced catabolic effects in ATDC5 cells and directly targets and inhibits MMP13 [17]. However, the effects of miR-320c on human chondrocyte inflammation are unclear. Using miRNA target prediction algorithms, we found that miR-320c regulates CDK6 expression and therefore hypothesized that miR320 c might play an important role in IL-1 $\beta$-induced human chondrocyte inflammation by regulating CDK6 expression. In this study, we investigated the roles of miR-320c and CDK6 in cartilage inflammation and determined their underlying mechanisms of action.

\section{Materials and Methods}

\section{Ethics}

All experimental procedures were approved by the ethical committee of The First Affiliated Hospital of Sun Yat-sen University (IRB: 2014C-028) and were performed in accordance with the Helsinki Declaration (2000). All volunteers included in the study provided written informed consent.

\section{Articular chondrocyte isolation and culture}

Human normal articular cartilage samples were obtained from nine patients (four men and five women; mean \pm SD age, $70.2 \pm 7.1$ years) who had no a history of OA or rheumatoi [20] d arthritis and who underwent hemiarthroplasty or total hip arthroplasty because of femoral neck fractures. Informed consent was obtained from all the patients. The cartilages were digested using $4 \mathrm{mg} / \mathrm{ml}$ protease and $0.25 \mathrm{mg} /$ $\mathrm{ml}$ collagenase $\mathrm{P}$, as described previously [20]. Cells obtained were cultured in DMEM/Nutrient Mixture F-12 (Gibco Life Technology, Grand Island, NY, USA) supplemented with 5\% fetal bovine serum (FBS; Gibco 


\section{Cellular Physiology Cell Physiol Biochem 2018;51:909-923

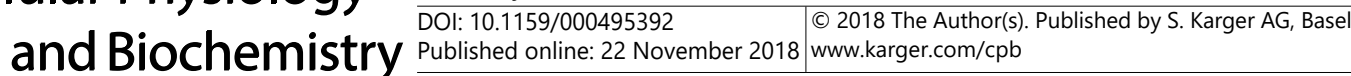 \\ Sun et al.: CDK6 and miR-320c Regulate Cartilage Inflammation}

Life Technology), $100 \mathrm{IU} / \mathrm{ml}$ penicillin, and $100 \mu \mathrm{g} / \mathrm{ml}$ streptomycin (Gibco Life Technology). Chondrocytes were used in experiments within 3-7 days and without passaging to prevent de-differentiation.

\section{Human mesenchymal stem cell isolation, culture, and chondrogenesis}

Bone marrow samples were obtained from three normal human donors (one man and two women; mean \pm SD age, $25.3 \pm 3.8$ years), and hBMSCs were isolated as described previously [20]. The cells were cultured in MSC basal medium (alpha-modified Eagle's medium [a-MEM]; Gibco Life Technology) supplemented with $10 \%$ FBS, $100 \mathrm{IU} / \mathrm{ml}$ penicillin, and $100 \mu \mathrm{g} / \mathrm{ml}$ streptomycin. Differentiation of hBMSCs into chondrocytes was induced by passaging the cells three times. hBMSC chondrogenesis was induced in a micromass culture, as described previously. For this, hBMSCs were resuspended in a-MEM supplemented with $10 \%$ FBS at a density of $10^{5}$ cells $/ \mu \mathrm{L}$, and $12.5 \mu \mathrm{L}$ cell suspension was dotted in the center of each well. The cells were incubated to stimulate their adherence to the plate. Next, the cells were incubated in a complete chondrogenic induction medium $(194 \mathrm{~mL}$ human mesenchymal stem cell chondrogenic differentiation basal medium; $20 \mu \mathrm{L}$ dexamethasone; $600 \mu \mathrm{L}$ ascorbate; $2 \mathrm{~mL}$ insulin, transferrin, and selenium supplement; $200 \mu \mathrm{L}$ sodium pyruvate; $200 \mu \mathrm{L}$ proline; and $2 \mathrm{ml}$ TGF- $\beta 3$; Cyagen, Guangzhou, China) or incomplete chondrogenic induction medium lacking TGF-b3 for $1 \mathrm{~h}$ at $37^{\circ} \mathrm{C}$ in a cell incubator to form a high-cell-density regions. At indicated time points, the cells were fixed in formalin and were stained with safranin $\mathrm{O}$ and alcian blue. In addition, the cells were used for performing immunohistochemical analysis of CDK6.

\section{Immunohistochemical and histological analyses}

Pregnant C57BL/6J mice were obtained from the animal center of Sun Yat-sen University, and forelimbs were harvested from mouse embryos at 16.5 days. Immunohistochemical analysis was performed as described previously [20]. Sections were treated with 3\% normal goat serum for $1 \mathrm{~h}$ and were incubated with specific primary antibodies against CDK6 (Abcam, RRID: AB_940952), SOX9 (Cell Signaling Technology, RRID: AB_2665492), MMP13 (Abcam, RRID: AB_1310459), and RUNX2 (Abcam, RRID: AB_943532), followed by incubation with a horseradish peroxidase (HRP)-conjugated anti-rabbit IgG secondary antibody (Cell Signaling Technology, RRID: AB_955447) for $30 \mathrm{~min}$. In addition, the sections were stained with safranin 0 and alcian blue.

\section{Transfection}

Normal and OA chondrocytes were transfected with $50 \mathrm{nM}$ hsa-miR-320c mimic or inhibitor (RiboBio, Guangzhou, China). In addition, normal chondrocytes were transfected with CDK6-siRNA or NC (RiboBio). All transfections were performed using Lipofectamine ${ }^{\circledR} 2000$ transfection reagent (Gibco Life Technologies), according to the manufacturer's instructions. Nonspecific microRNA (miR-control; RiboBio) was used as a control. The cells were harvested after $48 \mathrm{~h}$ for performing quantitative polymerase chain reaction (qPCR) and after $72 \mathrm{~h}$ for performing western blotting analysis.

RNA extraction, reverse transcription, and $q P C R$

Total cellular RNA was isolated using miRNeasy Mini Kit (Qiagen, Venlo, Netherlands). Next, cDNA was synthesized from mRNA and miRNA by using PrimeScript ${ }^{\mathrm{TM}}$ RT Master Mix (Takara, Shiga, Japan) and Mir- $\mathrm{X}^{\mathrm{TM}}$ miRNA FirstStrand Synthesis Kit (Clontech Laboratories, Inc., Mountain View, CA, USA), respectively. qPCR of miR-320c and target genes were performed using SYBR ${ }^{\circledR}$ Premix Ex Taq $^{\text {TM }}$ II (Takara) and KOD SYBR ${ }^{\circledR}$ qPCR Mix (Toyobo), according to the manufacturer's instructions, respectively. Transcript

Table 1. Primers for quantitative real-time polymerase chain reaction (qPCR)

\begin{tabular}{lcc}
\hline Gene & & Primer sequence (5'-3') \\
\hline hsa-miR-320c & F & AAAAGCAGGGAAGAGAGGGA \\
hsa-U6 & F & CTCGCTTCGGCAGCACA \\
hsa-U6 & R & AACGCTTCACGAATTTGCGT \\
Has-CDK6 & F & GCTGACCAGCAGTACGAATG \\
Hsa-CDK6 & R & GCACACATCAAACAACCTGACC \\
hsa-iNOS & F & TTCAGTATCACAACCTCAGCAAG \\
hsa-iNOS & R & TGGACCTGCAAGTTAAAATCCC \\
hsa-SOX9 & F & GGAGATGAAATCTGTTCTGGGAATG \\
hsa-SOX9 & R & TTGAAGGTTAACTGCTGGTGTTCTG \\
hsa-RUNX2 & F & CACTGGCGCTGCAACAAGA \\
hsa-RUNX2 & R & CATTCCGGAGCTCAGCAGAATAA \\
hsa-aggrecan & F & GATGTTCCCTGCAATTACCACCTC \\
hsa-aggrecan & R & TGATCTCATACCGGTCCTTCTTCTG \\
hsa-COL2A1 & F & GCACCTGCAGAGACCTGAAAC \\
hsa-COL2A1 & R & GCAAGTCTCGCCAGTCTCCA \\
hsa-MMP13 & F & TCCTGATGTGGGTGAATACAATG \\
hsa-MMP13 & R & TCCTGATGTGGGTGAATACAATG \\
hsa-GAPDH & F & GCACCGTCAAGGCTGAGAAC \\
hsa-GAPDH & R & TGGTGAAGACGCCAGTGGA \\
\hline
\end{tabular}

levels were normalized to those of the housekeeping 


\section{Cellular Physiology Cell Physiol Biochem 2018;51:909-923 \begin{tabular}{l|l|l} 
and Biochemistry Published online: 22 November 2018 & $\begin{array}{l}\text { ○ } 2018 \text { The Author(s). Published by S. Karger AG, Basel } \\
\text { www.karger.com/cpb }\end{array}$ \\
\hline
\end{tabular} \\ Sun et al.: CDK6 and miR-320c Regulate Cartilage Inflammation}

gene glyceraldehyde 3-phosphate dehydrogenase (GAPDH; for mRNA) or small U6 RNA (for miRNA). Sequences of specific primers used for these analyses are listed in Table 1. Gene expression was calculated using $2^{-\Delta \Delta C t}$ method, and each experiment was performed in triplicate.

\section{Western blotting analysis}

Western blotting analysis was performed as described previously [15]. Briefly, whole-cell extracts containing total cellular proteins were isolated from normal or OA chondrocytes by using RIPA buffer (Beyotime Biotechnology, Beijing, China) containing protease inhibitors (Abcam). Phosphatase inhibitors were used for detecting phosphorylated proteins. Next, $20 \mu \mathrm{g}$ proteins from each sample were resolved by performing sodium dodecyl sulfate-polyacrylamide gel electrophoresis and were transferred onto polyvinylidene difluoride membranes (Millipore, Bedford, MA, USA). The membranes were incubated with primary antibodies (dilution, 1:1000) against CDK6; SOX9; MMP13; NF- $\mathrm{B}$ signaling pathway proteins phosphorylated IKB $\alpha$ (Cell Signaling Technology, RRID:AB_561111), IKB $\alpha$ (Cell Signaling Technology, RRID:AB_390781), phosphorylated NF- $\kappa$ B p65 (Cell Signaling Technology, RRID:AB_331284), and NF- $\mathrm{BB}$ p65 (Cell Signaling Technology, RRID:AB_10859369); and MAPK signaling pathway proteins phosphorylated p38 (Cell Signaling Technology, RRID:AB_2139682), p38 (Cell Signaling Technology, RRID:AB_10999090), ERK1/2 (Cell Signaling Technology, RRID:AB_390779), phosphorylated ERK1/2 (Cell Signaling Technology, RRID:AB_2315112), SAPK/JNK (Cell Signaling Technology, RRID:AB_2250373), phosphorylated SAPK/JNK (Cell Signaling Technology, RRID:AB_823588). Next, the membranes were washed and were incubated with corresponding HRP-conjugated anti-mouse or anti-rabbit secondary antibodies (Cell Signaling Technology) for $1 \mathrm{~h}$ at room temperature. Protein bands were detected using an enhanced chemiluminescence detection system (GE Healthcare, Little Chalfont, UK), with GAPDH or tubulin as internal controls.

Luciferase constructs and reporter assay

DNA sequence of the CDK6 $3^{\prime}$-UTR was amplified by performing PCR with the following primers: 5'-ATAGGCCGGCATAGACGCGTGGCCTCAGCAGCCGC CTT-3' (forward) and 3'-AAAGATCCTTTATTAAGCTTGGATTTCAGAAA TGGCGAATC-5' (reverse). Amplified DNA sequences were inserted into pmiR-RB-REPORT ${ }^{\mathrm{TM}}$ vector (OBIO, Shanghai, China) to generate luciferase vectors expressing CDK6 3'-UTR or mutated CDK6 3'-UTR. For performing dual luciferase assay, $1.2 \times 10^{4}$ HEK293T cells seeded in a 96-well plate were transfected with $50 \mathrm{nM}$ hsa-miR-320c or mimic NC. Next, the cells were co-transfected with the $2 \mu \mathrm{g} / \mathrm{mL}$ vector expressing wild-type or mutant CDK6 3'-UTR. Luciferase activity was measured at $48 \mathrm{~h}$ after the transfection by using Dual-Luciferase ${ }^{\circledR}$ Reporter Assay System (Promega, Madison, WI, USA), according to the manufacturer's instructions.

\section{Cell viability assay}

Normal chondrocytes (8000 cells/well) were seeded into 96-well plates. After $24 \mathrm{~h}$, supernatant was removed and the cells were treated with abemaciclib or IL-1 $\beta$. Cell viability was measured at indicated time points by using Cell Counting Kit-8 (CCK-8; Dojindo Laboratories, Kumamoto, Japan), according to the manufacturer's instruction. Briefly, $10 \mu \mathrm{L}$ CCK-8 solution was added to each well, and the plates were incubated at $37^{\circ} \mathrm{C}$ for $2 \mathrm{~h}$. Absorbance was measured at $450 \mathrm{~nm}$ (OD $450 \mathrm{~nm}$ ) by using a microplate reader.

\section{Statistical analysis}

Statistical analyses were performed using GraphPad Prism software (GraphPad Prism Software, San Diego, CA). Data are presented as mean \pm standard deviation (SD) of the results of at least three independent experiments. Student's $t$-test and Mann-Whitney $U$ test were used to identify differences between groups, as appropriate. One-way analysis of variance and Kruskal-Wallis tests were used for performing multiple group comparisons. $\mathrm{P}<0.05$ was considered statistically significant for all the tests. 


\section{Cellular Physiology Cell Physiol Biochem 2018;51:909-923

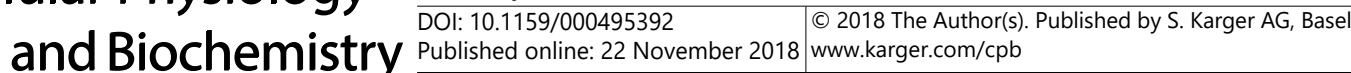 Sun et al.: CDK6 and miR-320c Regulate Cartilage Inflammation}

\section{Results}

Expression patterns of miR-320c, CDK6, COL2A1, and SOX9 during hBMSC chondrogenesis Expression patterns of miR-320c, CDK6, COL2A1, and SOX9 during chondrogenesis were determined by inducing the differentiation of hBMSCs into chondrocytes in micromass culture in vitro. We observed that expression of miR-320c in chondrogenic hBMSCs rapidly increased from day 7 onward, peaked on day 21, and markedly decreased on days 28 and 35 (Fig. 1a). Moreover, CDK6 expression showed concurrent significant upregulation between days 21 and 35 (Fig. 1b), suggesting that miR-320c affects CDK6 expression. Expression of SOX9 and COL2A1, markers of hBMSC chondrogenic differentiation, were similar to that of miR-320c throughout the experimental period (Fig. 1c, d). Results of immunohistochemical analysis also showed that CDK6 expression increased from days 3 to 7, decreased to its lowest level on day 21, and increased again from days 21 to 35 (Fig. 1e). The negative control of CDK6 staining on day 14 to day 28 is shown in Fig. 1f.

CDK6 expression during cartilage development

A previous study reported that proliferating, prehypertrophic, and hypertrophic chondrocytes can be detected in mouse limbs at E16.5 [21]. Our previous study showed high miR-320c expression in developmental and normal cartilages and low miR-320c expression in degenerating and OA cartilages. Therefore, we isolated mouse forelimbs at E16.5 to characterize the expression patterns of CDK6, SOX9, RUNX2, and MMP13 and to perform HE, safranin 0, and alcian blue staining (Fig. 2a, 2g, 2h). SOX9 was used as a positive control for early-stage chondrogenesis, and MMP13 was used as a positive control for late-stage chondrocytes. We observed moderate CDK6 expression in proliferating and prehypertrophic chondrocytes and high CDK6 expression in hypertrophic chondrocytes (Fig. 2b, c). A similar expression pattern was observed for MMP13 (Fig. 2e) and RUNX2 (Fig. 2f). Moreover, high expression of SOX9, the positive control for early-stage chondrogenesis, was detected in proliferating and pre-hypertrophic chondrocytes (Fig. $2 \mathrm{~d}$ ). The negative control of IHC staining is shown in Fig. 2i.

\section{miR-320c regulates CDK6 expression in normal chondrocytes}

To investigate whether miR-320c regulates CDK6 expression in normal chondrocytes, we suppressed or overexpressed miR-320c in chondrocytes. Normal chondrocytes were transfected with anti-miR-320c or miR-320c, and CDK6 and SOX9 expression levels were determined via qPCR and Western blotting. Treatment with miR-320c mimic significantly decreased CDK6 mRNA expression and increased SOX9 mRNA expression (Fig. 3a, b, e). In contrast, miR-320c inhibition significantly increased CDK6 expression and decreased SOX9 expression (Fig. 3c, d, e).

CDK6 modulates the expression of endogenous miR-320c in normal chondrocytes

To assess whether CDK6 modulates miR-320c expression in normal chondrocytes, we transfected chondrocytes with CDK6-siRNA. miR-320c expression was assessed by performing qPCR, and CDK6 expression was assessed via qPCR and Western blotting. Transfection of normal chondrocytes with CDK6-siRNA significantly decreased CDK6 and MMP13 expression (Fig. 4a, b, d) and increased miR-320c, COL2A1, and SOX9 expression (Fig. 4c, d).

miR-320c directly targets CDK6 by interacting with the 3'-UTR of its $m R N A$

Results of TargetScan analysis identified CDK6 as one of the three potential targets of hsamiR-320c (Fig. 5a). Therefore, we performed a luciferase reporter assay to examine whether miR-320c interacted with the $3^{\prime}$-UTR of CDK6 mRNA. We mutated three potential target sites in the CDK6 3'-UTR, and luciferase reporter assays were performed by transfecting cells with the luciferase vector expressing the CDK6 3'-UTR or one of the three mutated CDK6 3'UTR in the presence or absence of miR-320c overexpression. Cells co-transfected with the 


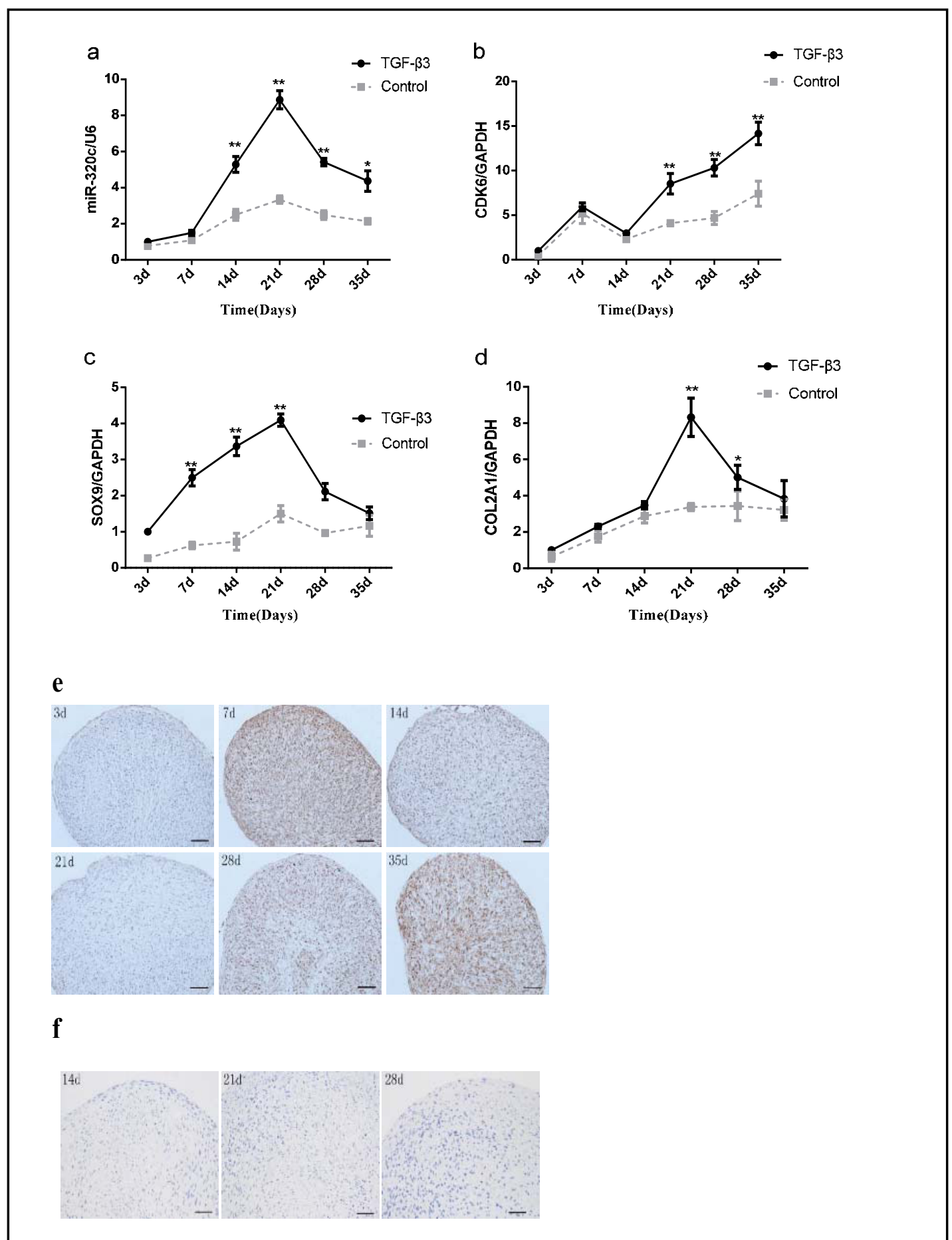

Fig. 1. Analysis of miR-320c and cyclin-dependent kinases 6 (CDK6) expression during chondrogenesis. hBMSCs cultured in chondrogenic differentiation medium for $0,7,14,21,28$ and 35 days. The expression of miR-320c (a), CDK6 (b), SOX9 (c), and COL2A1 (d) were detected by qPCR. hBMSCs cultured without TGF- $\beta 3$ at corresponding time points served as negative controls. U6 and GAPDH expression levels were measured and used as internal controls for microRNA and mRNA expression, respectively. Data are presented as means $\pm \mathrm{SD}$ of the results obtained from triplicate samples. ${ }^{*} \mathrm{P}<0.05 ;{ }^{* *} \mathrm{P}<0.001$ vs. negative controls, respectively. hBMSCs cultured with TGF- $\beta 3$ were stained with immunohistochemistry of CDK6 (e). (f) The IHC staining without first antibody was performed on day 14, 21, and 28 of chondrogenic differentiation, scale bar, 100 $\mu \mathrm{m}$.

\section{KARGER}




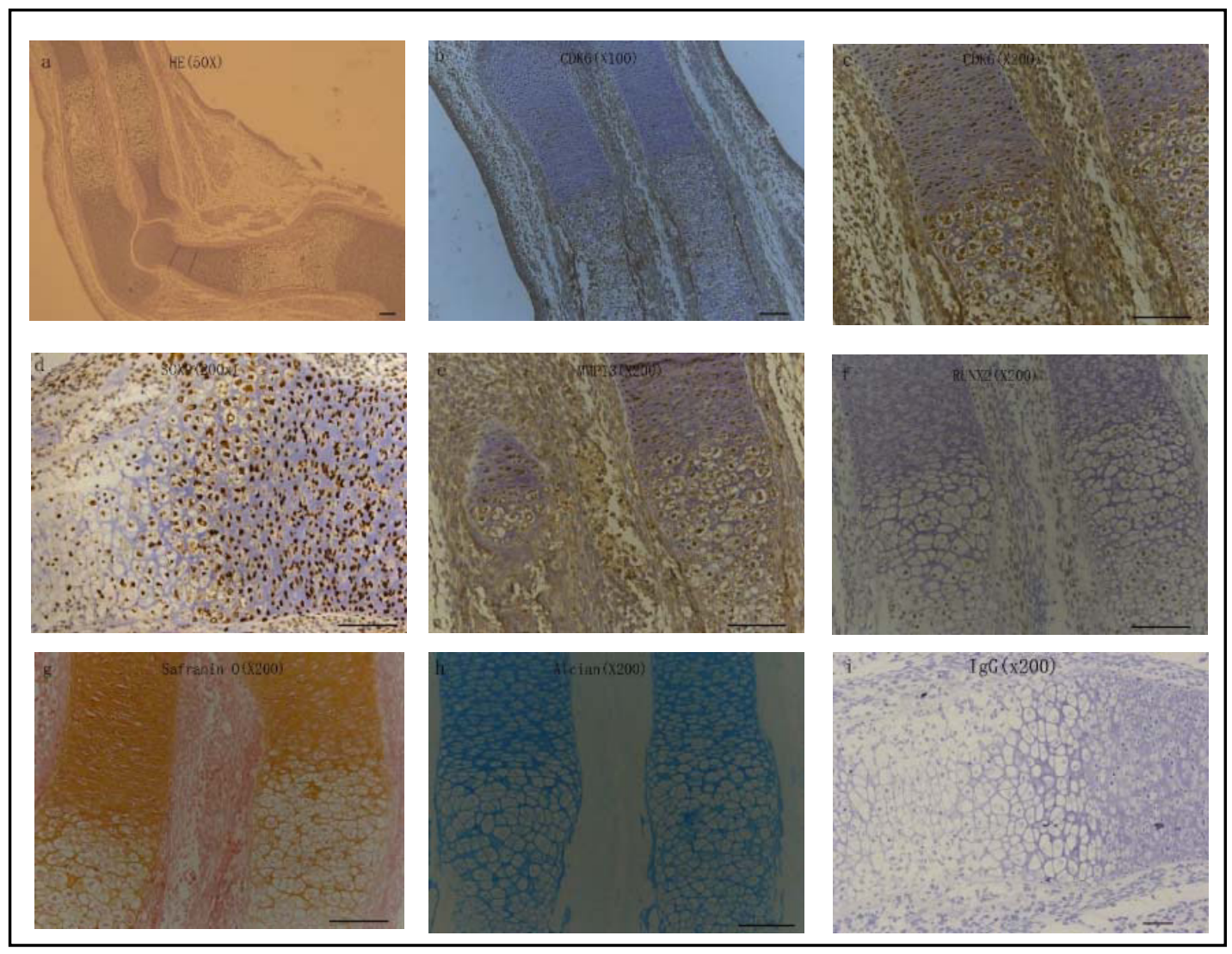

Fig. 2. The expression of CDK6 in forelimbs of mouse embryos at E16.5. HE strains in forelimbs of mouse embryos (a). The ulna and radius (b, c, e-h) or humerus $(d, i)$ in mouse embryos were stained with CDK6 (b, c), SOX9 (d), MMP13 (e), and RUNX2 (f) and IgG (i), or stained with safranin 0 (g) and alcian blue (h), scale bar, $100 \mu \mathrm{m}$.

CDK6 3'-UTR-expressing the luciferase reporter vector and miR-320c showed a significant decrease in luciferase activity (Fig. 5b). Mutation of the miR-320c binding sequence abolished the effect of miR-320c-mediated repression of the CDK6 3'-UTR luciferase vector (Fig. 5b). Together, these results indicate that miR-320c modulates $C D K 6$ expression by binding to the $3^{\prime}$-UTR of its mRNA and that CDK6 is a target of miR-320c.

miR-320c overexpression and CDK6 inhibition attenuate IL-1 $\beta$-induced inflammation in normal chondrocytes

Our previous study showed that mmu-miR-320 inhibited IL-1 $\beta$-induced catabolism in mouse chondrocytes [15]. Therefore, we investigated the effects of hsa-miR-320c and CDK6 on IL-1 $\beta$-induced inflammation in normal human chondrocytes. Chondrocytes were transfected with $50 \mathrm{nM}$ miR-320c or CDK6-siRNA, followed by stimulation with $5 \mathrm{ng} / \mathrm{ml} \mathrm{IL-}$ $1 \beta$ at $48 \mathrm{~h}$ after transfection. Total cellular RNA was extracted after $1,3,6,12$, and $24 \mathrm{~h}$ of IL-1 $\beta$ stimulation, while protein was isolated after $48 \mathrm{~h}$ of IL-1 $\beta$ stimulation. Expression levels of miR-320c, CDK6, MMP13, iNOS, COL2A1, and aggrecan were assessed via qPCR or Western blotting. Our results showed that miR-320c overexpression and CDK6 inhibition increased miR-320c levels (Fig. 6a, b) and that IL-1 $\beta$ stimulation decreased miR-320c levels in a time-dependent manner [Fig. 6a, b]. Moreover, miR-320c overexpression (Fig. 6c-f) and CDK6 inhibition (Fig. 6g-l) attenuated the decrease in COL2A1 and aggrecan expression and increase in iNOS and MMP13 expression. 


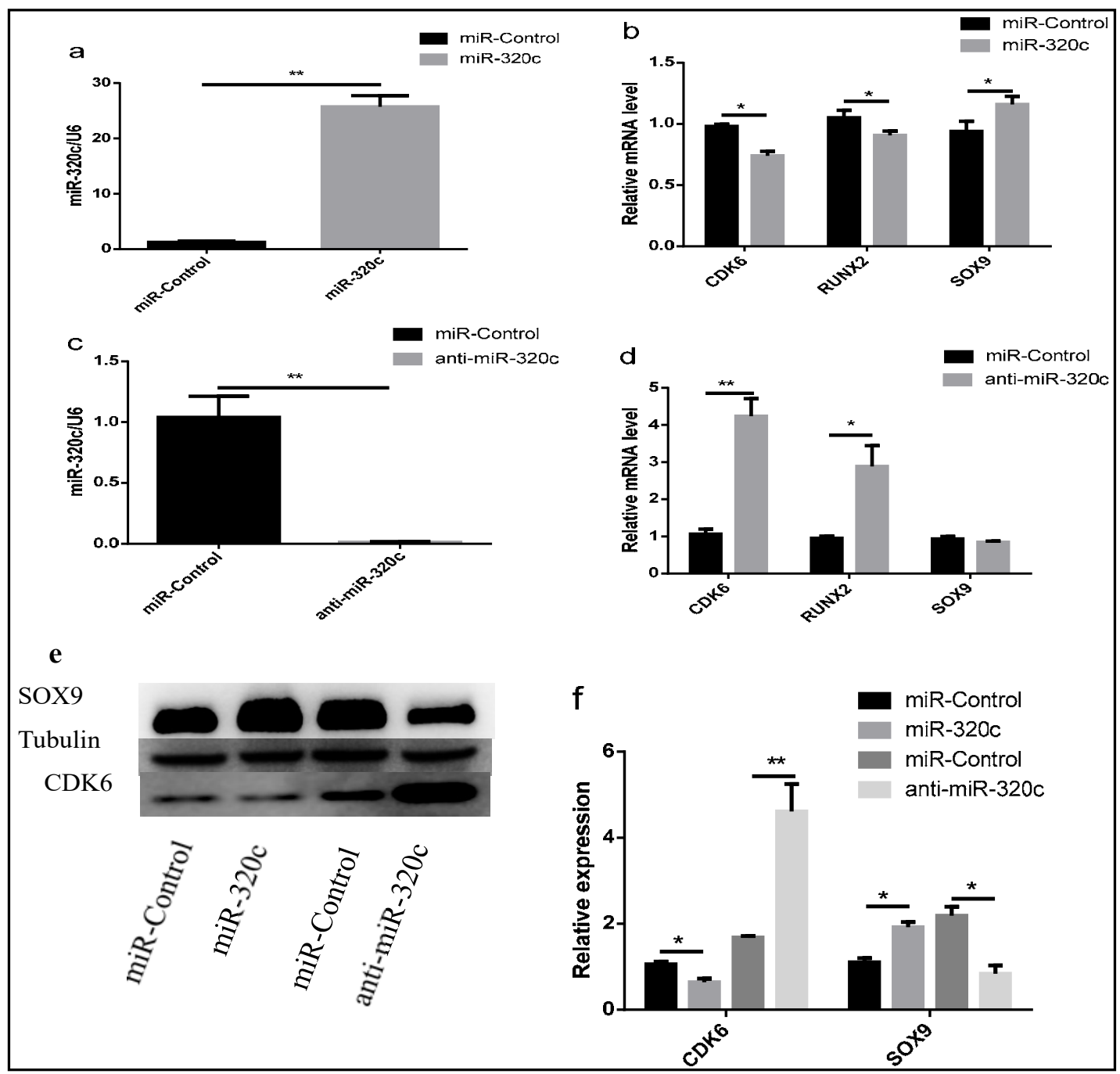

Fig. 3. miR-320c inhibits the expression of CDK6, and regulates RUNX2 and SOX9 expression. Normal chondrocytes were transfected with miR-320c mimic, a non-specific control (NC) mimic, miR-320c inhibitor, or an NC inhibitor. The effect of inhibited (a,b) or overexpressed (c,d) miR-320c on CDK6, RUNX2, and SOX9 were detected by qPCR. Data are presented as means \pm SD of the results of three independent experiments. ${ }^{*} \mathrm{P}<0.05$, ${ }^{* *} \mathrm{P}<0.001$. Western blot analysis of CDK6 and SOX9 expression levels (e), and quantification (f). GAPDH or Tubulin expression levels were measured and used as internal controls. The experiment was performed in triplicate and a representative image is shown.

Abemaciclib attenuates $I L-1 \beta$-induced inflammation in chondrocytes through the NF-kB and MAPK signaling pathways

Previous studies have shown that CDK6 is not a traditional therapeutic target for treating OA. Therefore, we investigated the effect of abemaciclib, a selective CDK4 and CDK6 inhibitor, on IL-1 $\beta$-induced inflammation in chondrocytes and its possible underlying mechanism of action. Because there is no specific inhibitor of CDK6, we evaluated the inhibitory effect of CDK4 on IL-1 $\beta$ induced chondrocytes. Chondrocytes were transfected with $50 \mathrm{nM}$ CDK4siRNA and were stimulated with $5 \mathrm{ng} / \mathrm{ml} \mathrm{IL-1 \beta}$ after $48 \mathrm{~h}$ of transfection. Transfection of CDK4-siRNA slightly decreased MMP13 (Fig. 7a), indicating that abemaciclib inhibited IL$1 \beta$-induced inflammation in chondrocytes by primarily inhibiting CDK6. Next, we treated chondrocytes with differing concentrations of abemaciclib for $24 \mathrm{~h}$ to evaluate the effect of abemaciclib on cell viability. Because treatment with 5 and $10 \mathrm{nM}$ abemaciclib did not affect chondrocyte viability (Fig. 7b), we used these two concentrations for performing 


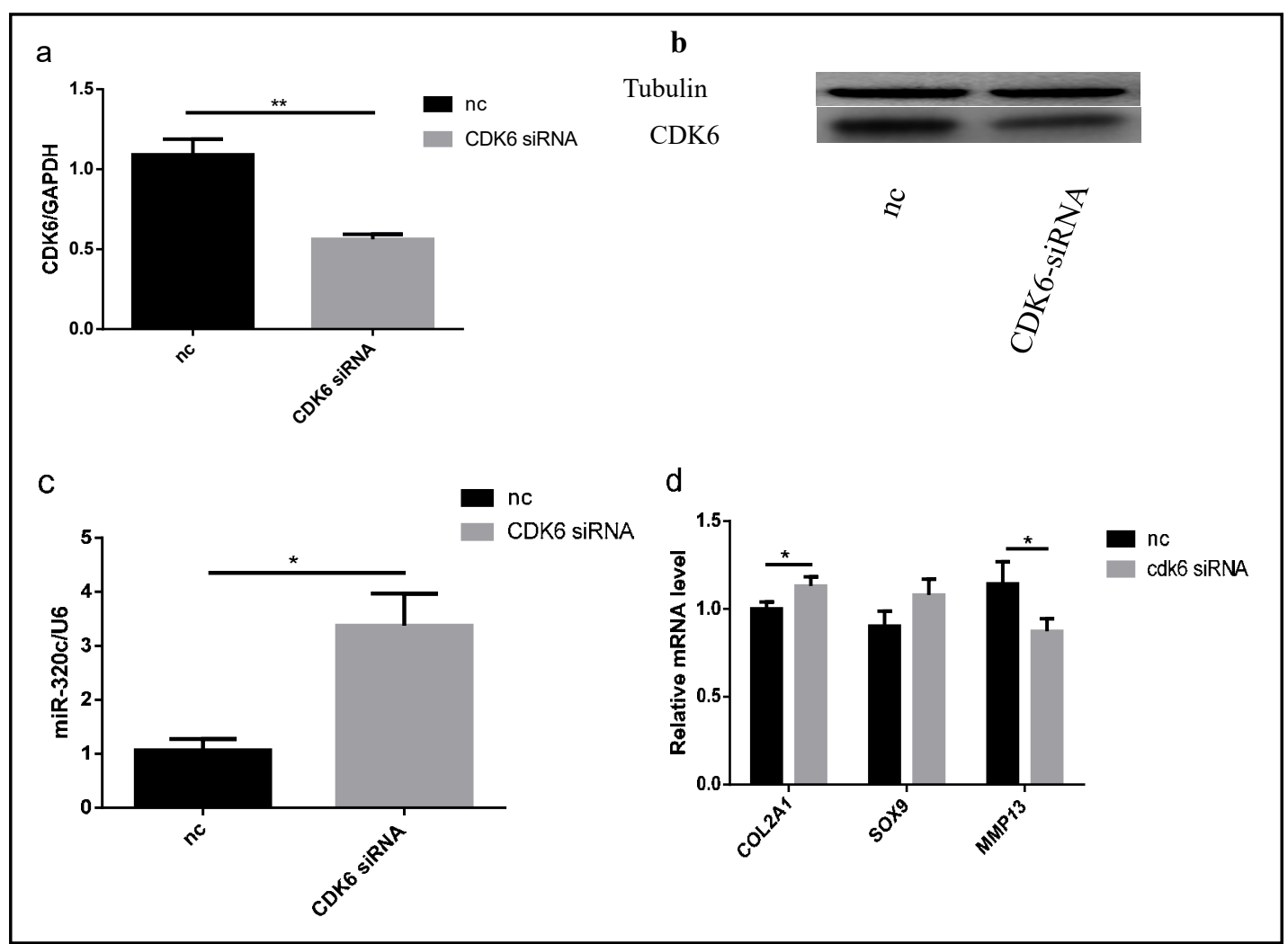

Fig. 4. CDK6 modulated expression of miR-320c in normal chondrocytes. Normal chondrocytes were transfected with CDK6 siRNA, or a non-specific control (NC) siRNA, respectively. The effect of inhibiting CDK6 were detected by qPCR (a) and western blot (b). CDK6 modulated expression of miR-320c (c), COL2A1, SOX9, and MMP13 (d). Data are presented as means \pm SD of the results of three independent experiments. ${ }^{*} \mathrm{P}<0.05,{ }^{* *} \mathrm{P}<0.001$. GAPDH expression levels were measured and used as internal controls. The experiment was performed in triplicate and a representative image is shown.

Fig. 5. miR-320c directly targets CDK6. Alignment of the nucleotide sequence of miR-320c and the $3^{\prime}$-UTR CDK6 mRNA (a). A luciferase reporter carrying the 3'-UTR of CDK6 or mutant CDK6 in which the binding site of miR-320c was mutated (Luc-CDK6-UTR-mut) was introduced into 293T cells along with negative miR-control (miR-Control), miR-320c, respectively. The cells were harvested $48 \mathrm{~h}$ later for luciferase assays (b). Data are presented as means \pm SD of the results of three independent experiments. ${ }^{*} \mathrm{P}<0.05,{ }^{* *} \mathrm{P}<0.001$.

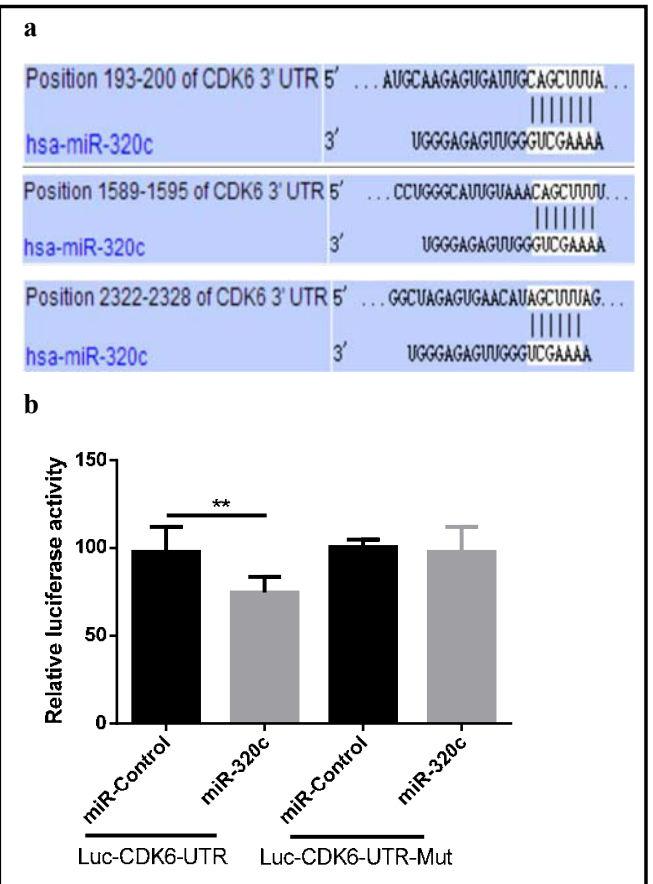


Fig. 6. Overexpression of miR-320c and inhibition of CDK6 attenuate IL-1 $\beta$ induced inflammation. $\begin{array}{llllll}\mathrm{N} & \mathrm{o} & \mathrm{r} & \mathrm{m} & \mathrm{a} & \mathrm{l}\end{array}$ chondrocytes were transfected with miR-320c mimic, or CDK6 siRNA, and then stimulated with IL- $1 \beta$ by $5 \mathrm{ng} /$ $\mathrm{ml}$ for $0,1,3$, $6,12,24 \mathrm{~h}$. The expression of miR-320c (a,b), iNOS (c,g), M M P $13(\mathrm{~d}, \mathrm{~h})$, COL2A1 (e,i), and Aggrecan

were detected by qPCR or western blot. The protein was isolated after $48 \mathrm{~h}$ of IL$1 \beta$ stimulation, and then MMP13 and CDK6 were detected by western blot (k), and quantification (l). Data are presented as means \pm SD of the results of three i n d e p end e n t experiments. Nc, normal control, ${ }^{*} \mathrm{P}<0.05$, $* * \mathrm{P}<0.001$. GAPDH and Tubulin expression levels were measured and used as internal controls for qRT-PCR and western blot, respectively. The experiment was performed in triplicate and a representative image is shown.
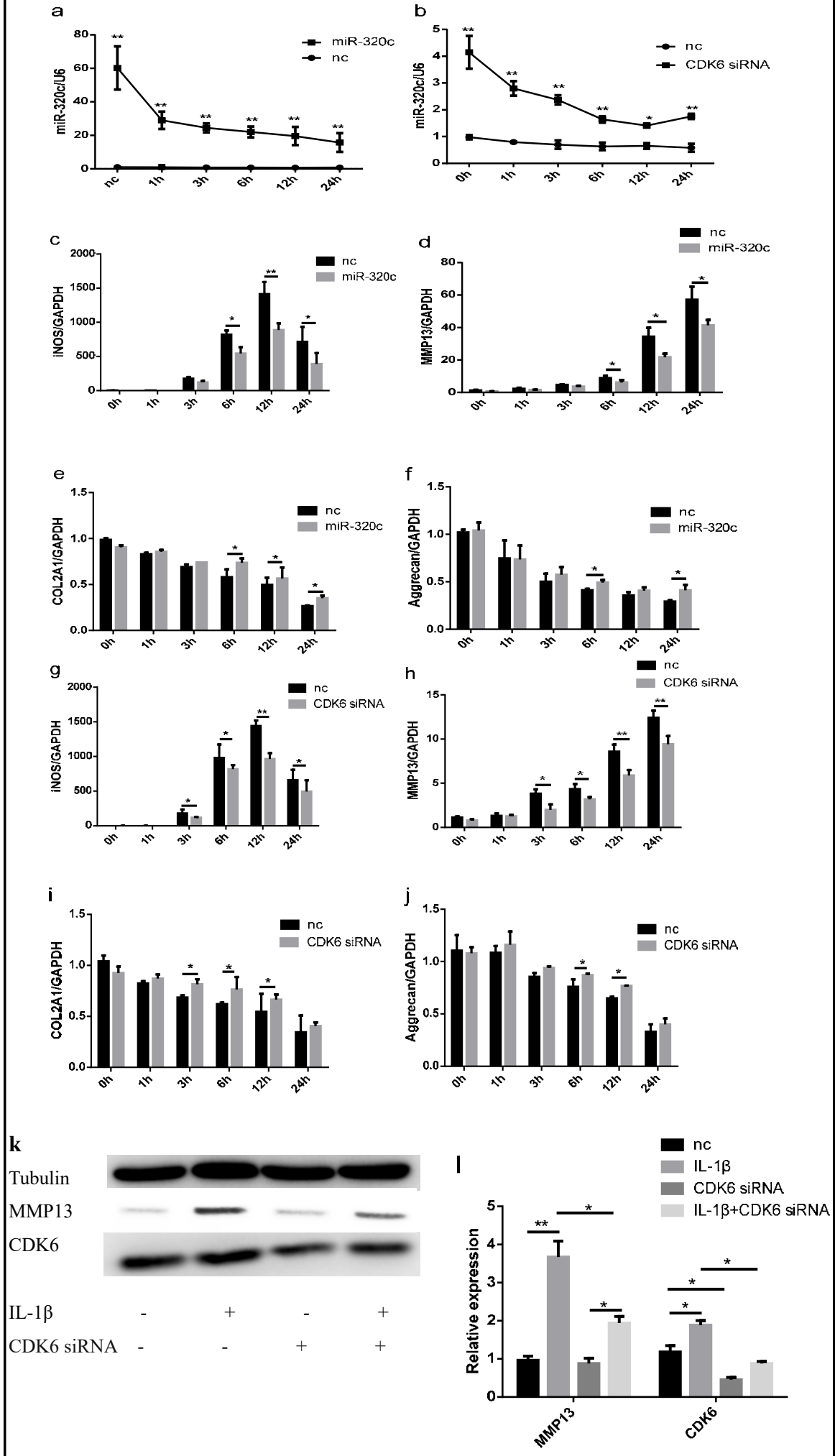


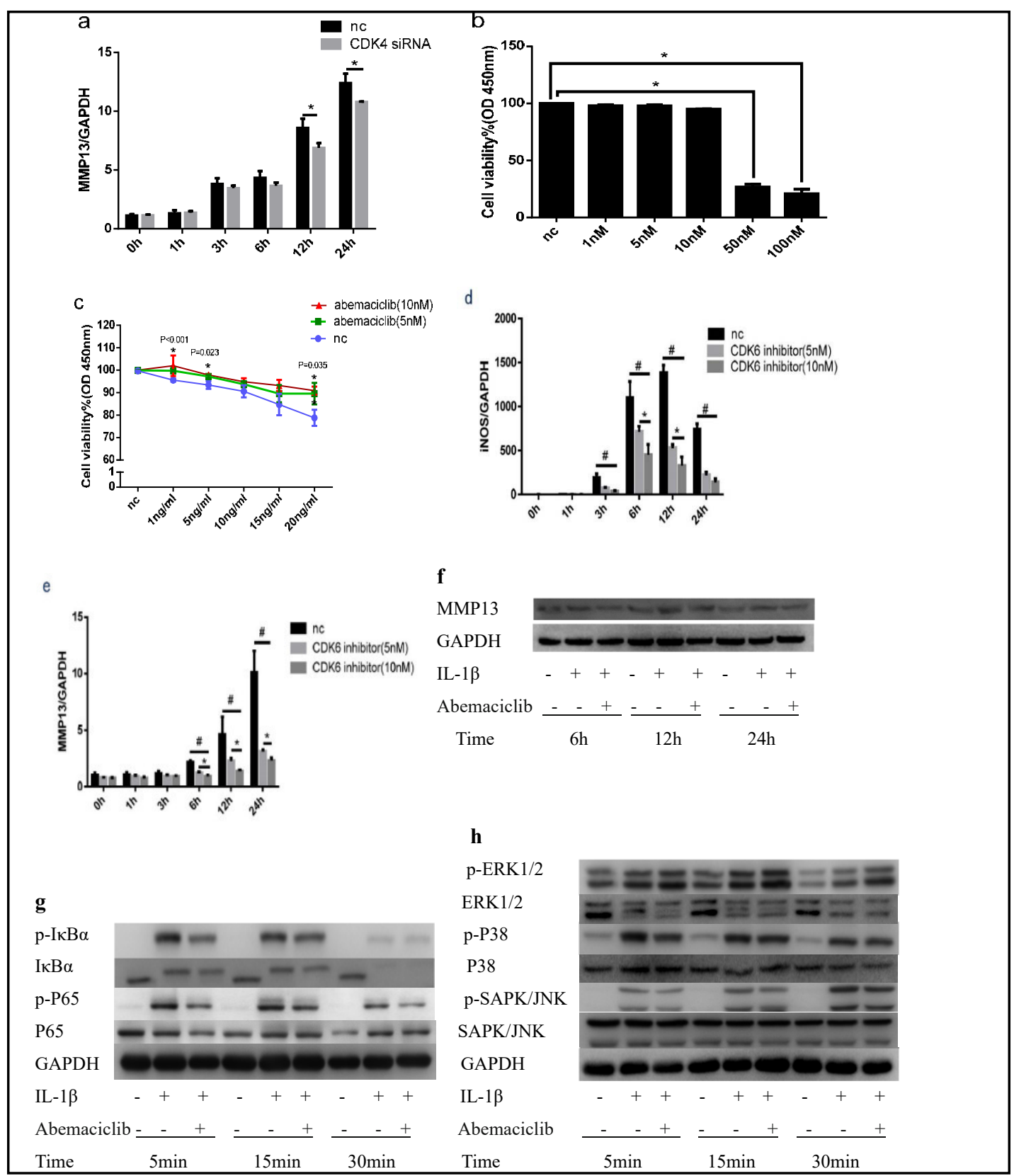

Fig. 7. Abemaciclib attenuated IL-1 $\beta$ induced chondrocytes inflammation response. The expression of MMP13 in IL-1 $\beta$ induced chondrocytes inflammation response after the transfection of CDK4 (a). Cell viability of normal chondrocytes treated with 0-100 nM abemaciclib (b). Normal chondrocytes were treated with $5 \mathrm{ng} / \mathrm{ml}$ IL-1 $\beta$ and abemaciclib in different concentrations $(0 \mathrm{nM}, 5 \mathrm{nM}, 10 \mathrm{nM})$ for $24 \mathrm{~h}$, and then cell viability (c) and mRNA level of iNOS (d), MMP13 (e) were detected. Normal chondrocytes were treated with $5 \mathrm{ng} / \mathrm{ml} \mathrm{IL-1 \beta}$ and $10 \mathrm{nM}$ abemaciclib for $0,3,6,12,24 \mathrm{~h}$, and the expression of MMP13 were detected by western blot (f). Normal chondrocytes were treated with $5 \mathrm{ng} / \mathrm{ml} \mathrm{IL-1 \beta}$ and $10 \mathrm{nM}$ abemaciclib for 5 , $15,30 \mathrm{~min}$, and the phosphorylation of $\mathrm{p} 65, \mathrm{IKB} \alpha(\mathrm{g})$, and the phosphorylation of p38, ERK1/2, JNK/SAPK were detected by western blot $(\mathrm{h})$. Data are presented as means \pm SD of the results of three independent experiments. ${ }^{*} \mathrm{P}<0.05,{ }^{* *} \mathrm{P}<0.001$, \# Group nc compared with abemaciclib $5 \mathrm{nM}$ and $10 \mathrm{nM}$ have significant difference. GAPDH expression levels were measured and used as internal controls. The experiment was performed in triplicate and a representative image is shown. 


\section{Cellular Physiology Cell Physiol Biochem 2018;51:909-923

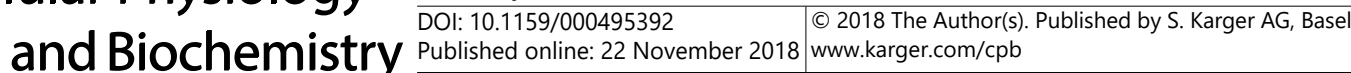 \\ Sun et al.: CDK6 and miR-320c Regulate Cartilage Inflammation}

subsequent experiments. Next, we treated chondrocytes with $5 \mathrm{ng} / \mathrm{ml} \mathrm{IL-1 \beta}$ and different concentrations $(0,5$, and $10 \mathrm{nM})$ of abemaciclib for $24 \mathrm{~h}$ and determined changes in cell viability. Treatment with both 5 and $10 \mathrm{nM}$ abemaciclib weakened the effect of IL1- $\beta$ on cell viability (Fig. 7c); however, the difference between the effect of these two concentrations of abemaciclib was not statistically significant. Abemaciclib also alleviated the $5 \mathrm{ng} / \mathrm{ml} \mathrm{IL-1 \beta -}$ induced increase in MMP13 and iNOS levels; however, this effect was more pronounced with $10 \mathrm{nM}$ abemaciclib than with $5 \mathrm{nM}$ abemaciclib (Fig. 7d, e, f).

The NF- $\kappa B$ and MAPK signaling pathways play an important role in OA [22]. NF- $\kappa B$ has two active pathways; namely, the non-canonical pathway, which promotes the activation of NF- $\kappa \mathrm{B}$ p50 and p52 [23], and the canonical pathway, which is based on NF- $\mathrm{BB}$ p65/

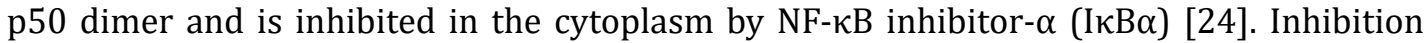
of IKB $\alpha$ phosphorylation inactivates the canonical NF- $\kappa B$ pathway $[25,26]$. Therefore, we investigated the effect of abemaciclib on the canonical NF- $\kappa B$ pathway in IL-1 $\beta$-induced chondrocytes. Chondrocytes were treated with $10 \mathrm{nM}$ abemaciclib and $5 \mathrm{ng} / \mathrm{ml} \mathrm{IL-1 \beta}$, and protein was extracted at 5,15 , and 30 min after IL-1 $\beta$ stimulation. Levels of phosphorylated IKB $\alpha$, unphosphorylated IKB $\alpha$, phosphorylated NF- $\kappa B$ p65, and unphosphorylated NF$\kappa \mathrm{B}$ p65 were assessed by performing western blotting. Abemaciclib attenuated the phosphorylation of IKB $\alpha$ and NF- $\mathrm{KB}$ p65, indicating that it functioned as a negative regulator of NF-kB signaling (Fig. 7g). Proteins associated with the MAPK signaling pathway play an important role in inflammatory responses in chondrocytes. Inhibition of p38 and SAPK/JNK suppresses apoptosis and the production of proinflammatory cytokines [27, 28]. Therefore, we investigated whether the selective CDK6 inhibitor regulates MAPK signaling by determining the levels of phosphorylated p38, phosphorylated ERK1/2, and phosphorylated SAPK/JNK by performing Western blotting analysis. Our results showed that abemaciclib did not significantly alter the phosphorylation of p38, SAPK/JNK, or ERK1/2 (Fig. 7h).

\section{Discussion}

OA develops as a result of an imbalance between anabolic and catabolic processes. The present study is the first to show that CDK6 and miR-320c co-regulate chondrogenesis and IL-1 $\beta$-induced chondrocyte inflammation. Our results show that miR-320c regulates CDK6 expression and that CDK6 modulates miR-320c expression in return. miR-320c overexpression and CDK6 inhibition also repressed IL-1 $\beta$-induced inflammation in chondrocytes by regulating the NF-kB signaling pathway. Considering these results of CDK6 and miR-320c expression and regulation during chondrogenesis of mesenchymal stem cells and chondrocyte inflammation, the co-regulation of CDK6 and miR-320c could potentially alter chondrogenic differentiation of MSCs and osteoarthritis.

In recent years, miRNAs have gained increasing importance due to their regulation of the expression of various genes, including cartilage-specific genes [15-17, 20]. miR-320c specifically has been shown to promote embryonic development in mice [29] and regulate hBMSC adipocytic differentiation by directly targeting RUNX2 [30]. A previous study also showed that miR-320c regulates chondrogenesis and IL-1 $\beta$-induced inflammation in murine ATDC5 cells [17]. Our study similarly shows that miR-320c expression increased in the developmental stage and decreased in the degeneration stage of hBMSC chondrogenic differentiation.

Moreover, our results showed that hsa-miR-320c targeted the 3'-UTR of human CDK6 mRNA and downregulated its expression. CDKs perform various functions by regulating the cell cycle and gene expression, indicating their involvement in diverse biological processes [31]. miR-107 and miR-186 directly regulate CDK6 expression by interacting with the $3^{\prime}-$ UTR of its mRNA to inhibit cancer cell proliferation $[32,33]$. Elizabeth et al. demonstrated that Toll-like Receptor-4 (TLR4) down-regulated miR-107, thus increasing CDK6 expression and promoting macrophage adhesion [32]. However, it has been shown that individual or combined deletion of $C D K 4$ and CDK6 does not affect the proliferation of normal cells [9, 
10]. We likewise observed that treatment with moderate concentrations of CDK4 and CDK6 inhibitors did not affect chondrocyte viability. Interestingly, Trohatou et al. showed that miR26a-induced CDK6 inhibition enhanced the adipogenic differentiation of hBMSC [34].

CDK6 has been shown to regulate inflammatory responses. $\mathrm{P} 21^{\mathrm{CIP}} 1$ inhibits the activity of CDK4/CDK6, and P21 ${ }^{C I P 1}$ deletion increases CDK6 expression and NF-kB activity [35]. Moreover, production of proinflammatory mediators such as TNF- $\alpha$ and IL- $1 \beta$ enhanced [36]. CDK6 interacts with the NF- $\kappa B$ transcription factor $\mathrm{p} 65$ and the promoters of many transcriptionally active NF- $\kappa \mathrm{B}$-target genes, such as thyroid hormone receptor-interacting protein 6 gene [37]. Buss et al. found that CDK6 promotes p65 phosphorylation to regulate inflammatory gene expression [12]. Our study showed that miR-320c overexpression and CDK6 inhibition attenuated IL-1 $\beta$-induced increases in expression of inflammatory products such as iNOS and MMP13 and decreased the expression of anabolic products such as COL2A1 and aggrecan. Moreover, we found that treatment with a moderate concentration of abemaciclib, a CDK4/CDK6 inhibitor, alleviated IL-1 $\beta$-induced increases in MMP13 and iNOS expression in normal chondrocytes.

The NF-kB and MAPK signaling pathways play an important role in OA development

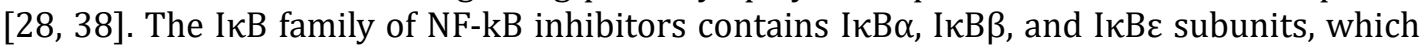
undergo proteasomal degradation after their phosphorylation. I $\kappa \varepsilon \varepsilon$ undergoes slow degradation, whereas I $\mathrm{K} \mathrm{B} \alpha$ undergoes rapid degradation after inflammatory stimulation $[39,40]$. Our findings indicate that abemaciclib functions as a negative regulator of NF-kB signaling and represses $I \kappa B \alpha$ and NF- $\kappa B$ p 65 phosphorylation, thus inhibiting the expression of downstream inflammatory products such as iNOS. The MAPK signaling pathway regulates many physiological and pathological processes in eukaryotic cells, including inflammation, development, and apoptosis. A previous study showed that p38 and SAPK/JNK regulate inflammation in chondrocytes. However, our results showed that CDK6 inhibition did not alter the phosphorylation of $\mathrm{p} 38$, SAPK/JNK, and ERK1/2, suggesting that the effect of CDK6 inhibition on inflammation repression is not via the MAPK signaling pathway.

\section{Conclusion}

In summary, the results of this study indicate that miR-320c and CDK6 co-regulate hBMSC chondrogenesis and IL-1 $\beta$-induced chondrocyte inflammation. Increased miR-320c expression and decreased CDK6 expression repress the activation of the NF-kB pathway, thus attenuating the expression of inflammatory products such as iNOS and regulating inflammation and cell viability. To the best of our knowledge, this is the first study to show that CDK6 and a miRNA co-regulate hBMSC chondrogenesis and chondrocyte inflammation. These results not only provide new insights into the regulation of chondrogenesis and inflammation by CDK6 and miR-320c but also highlight the possibility of using miR-320c and CDK6 inhibitors to repress catabolism in human chondrocytes.

\section{Acknowledgements}

This study was funded by the National Natural Science Foundation of China (no. 81472101, 81672145, and 81572119), the Science and Technology Project of Guangdong Province (2014A020211003 and 2015A020212015) and the Science and Technology Project of Guangzhou City (201710010164). We thank Aaron Weiner from University of Pennsylvania for English revision.

\section{Disclosure Statement}

The authors declare that they do not have any conflicts of interest related to this study.

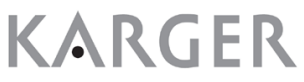




\section{Cellular Physiology Cell Physiol Biochem 2018;51:909-923 and Biochemistry \begin{tabular}{l|l} 
DOI: 10.1159/000495392 2018 The Author(s). Published by S. Karger AG, Basel \\
Publion
\end{tabular}

\section{References}

1 Kapoor M, Martel-Pelletier J, Lajeunesse D, Pelletier JP, Fahmi H: Role of proinflammatory cytokines in the pathophysiology of osteoarthritis. Nat Rev Rheumatol 2011;7:33-42.

-2 Yang P, Tan J, Yuan Z, Meng G, Bi L, Liu J: Expression profile of cytokines and chemokines in osteoarthritis patients: Proinflammatory roles for CXCL8 and CXCL11 to chondrocytes. Int Immunopharmacol 2016;40:16-23.

-3 Goldring MB, Marcu KB: Cartilage homeostasis in health and rheumatic diseases. Arthritis Res Ther 2009;11:224.

4 Hsu YH, Yang YY, Huwang MH, Weng YH, Jou IM, Wu PT, Lin TY, Wu LW, Chang MS: Anti-IL-20 monoclonal antibody inhibited inflammation and protected against cartilage destruction in murine models of osteoarthritis. PLoS One 2017;12:e0175802.

-5 Wang AZ, Wang JC, Fisher GW, Diamond HS: Interleukin-1beta-stimulated invasion of articular cartilage by rheumatoid synovial fibroblasts is inhibited by antibodies to specific integrin receptors and by collagenase inhibitors. Arthritis Rheum 1997;40:1298-1307.

6 Yaron M, Shirazi I, Yaron I: Anti-interleukin-1 effects of diacerein and rhein in human osteoarthritic synovial tissue and cartilage cultures. Osteoarthritis Cartilage 1999;7:272-280.

7 Dickler MN, Tolaney SM, Rugo HS, Cortes J, Dieras V, Patt D, Wildiers H, Hudis CA, O'Shaughnessy J, Zamora E, Yardley DA, Frenzel M, Koustenis A, Baselga J: MONARCH 1, A Phase II Study of Abemaciclib, a CDK4 and CDK6 Inhibitor, as a Single Agent, in Patients with Refractory HR(+)/HER2(-) Metastatic Breast Cancer. Clin Cancer Res 2017;23:5218-5224.

-8 Ventz S, Alexander BM, Parmigiani G, Gelber RD, Trippa L: Designing Clinical Trials That Accept New Arms: An Example in Metastatic Breast Cancer. J Clin Oncol 2017;35:3160-3168.

9 Malumbres M, Barbacid M: Cell cycle, CDKs and cancer: a changing paradigm. Nat Rev Cancer 2009;9:153166.

10 Malumbres M, Sotillo R, Santamaria D, Galan J, Cerezo A, Ortega S, Dubus P, Barbacid M: Mammalian cells cycle without the D-type cyclin-dependent kinases Cdk4 and Cdk6. Cell 2004;118:493-504.

11 Bockstaele L, Bisteau X, Paternot S, Roger PP: Differential regulation of cyclin-dependent kinase 4 (CDK4) and CDK6, evidence that CDK4 might not be activated by CDK7, and design of a CDK6 activating mutation. Mol Cell Biol 2009;29:4188-4200.

12 Buss H, Handschick K, Jurrmann N, Pekkonen P, Beuerlein K, Muller H, Wait R, Saklatvala J, Ojala PM, Schmitz ML, Naumann M, Kracht M: Cyclin-dependent kinase 6 phosphorylates NF-kappaB P65 at serine 536 and contributes to the regulation of inflammatory gene expression. PLoS One 2012;7:e51847.

-13 Yik JH, Hu Z, Kumari R, Christiansen BA, Haudenschild DR: Cyclin-dependent kinase 9 inhibition protects cartilage from the catabolic effects of proinflammatory cytokines. Arthritis Rheumatol 2014;66:1537-1546.

14 Bartel DP: MicroRNAs: genomics, biogenesis, mechanism, and function. Cell 2004;116:281-297.

15 Chen W, Chen L, Zhang Z, Meng F, Huang G, Sheng P, Zhang Z, Liao W: MicroRNA-455-3p modulates cartilage development and degeneration through modification of histone H3 acetylation. Biochim Biophys Acta 2016;1863:2881-2891.

16 Hou C, Meng F, Zhang Z, Kang Y, Chen W, Huang G, Fu M, Sheng P, Zhang Z, Liao W: The Role of MicroRNA-381 in Chondrogenesis and Interleukin-1-beta Induced Chondrocyte Responses. Cell Physiol Biochem 2015;36:1753-1766.

17 Meng F, Zhang Z, Chen W, Huang G, He A, Hou C, Long Y, Yang Z, Zhang Z, Liao W: MicroRNA-320 regulates matrix metalloproteinase-13 expression in chondrogenesis and interleukin-1beta-induced chondrocyte responses. Osteoarthritis Cartilage 2016;24:932-941.

18 Pierdomenico M, Cesi V, Cucchiara S, Vitali R, Prete E, Costanzo M, Aloi M, Oliva S, Stronati L: NOD2 Is Regulated By Mir-320 in Physiological Conditions but this Control Is Altered in Inflamed Tissues of Patients with Inflammatory Bowel Disease. Inflamm Bowel Dis 2016;22:315-326.

19 Ukai T, Sato M, Akutsu H, Umezawa A, Mochida J: MicroRNA-199a-3p, microRNA-193b, and microRNA-320c are correlated to aging and regulate human cartilage metabolism. J Orthop Res 2012;30:1915-1922.

-20 Mao G, Zhang Z, Huang Z, Chen W, Huang G, Meng F, Zhang Z, Kang Y: MicroRNA-92a-3p regulates the expression of cartilage-specific genes by directly targeting histone deacetylase 2 in chondrogenesis and degradation. Osteoarthritis Cartilage 2017;25:521-532. 


\section{Cellular Physiology Cell Physiol Biochem 2018;51:909-923 \begin{tabular}{l|l|l|l|l|}
\hline DOI: 10.1159/000495392 2018 The Author(s). Published by S. Karger AG, Basel & and Biochemistry
\end{tabular}

-21 Hsu CC, Lien JC, Chang CW, Chang CH, Kuo SC, Huang TF: Yuwen02f1 suppresses LPS-induced endotoxemia and adjuvant-induced arthritis primarily through blockade of ROS formation, NFkB and MAPK activation. Biochem Pharmacol 2013;85:385-395.

-22 Hacker H, Karin M: Is NF-kappaB2/p100 a direct activator of programmed cell death? Cancer Cell 2002;2:431-433.

23 Moskowitz RW: Osteoarthritis cartilage histopathology: grading and staging. Osteoarthritis Cartilage 2006;14:1-2.

24 Hinz M, Arslan SC, Scheidereit C: It takes two to tango: IkappaBs, the multifunctional partners of NFkappaB. Immunol Rev 2012;246:59-76.

-25 Liu B, Sun L, Liu Q Gong C, Yao Y, Lv X, Lin L, Yao H, Su F, Li D, Zeng M, Song E: A cytoplasmic NF-kappaB interacting long noncoding RNA blocks IkappaB phosphorylation and suppresses breast cancer metastasis. Cancer Cell 2015;27:370-381.

-26 Morotti A, Crivellaro S, Panuzzo C, Carra G, Guerrasio A, Saglio G: IkappaB-alpha: At the crossroad between oncogenic and tumor-suppressive signals. Oncol Lett 2017;13:531-534.

27 Kitanaka T, Nakano R, Kitanaka N, Kimura T, Okabayashi K, Narita T, Sugiya H: JNK activation is essential for activation of MEK/ERK signaling in IL-1beta-induced COX-2 expression in synovial fibroblasts. Sci Rep 2017;7:39914.

28 Sun HY, Hu KZ, Yin ZS: Inhibition of the p38-MAPK signaling pathway suppresses the apoptosis and expression of proinflammatory cytokines in human osteoarthritis chondrocytes. Cytokine 2017;90:135143.

29 Feng R, Sang Q, Zhu Y, Fu W, Liu M, Xu Y, Shi H, Xu Y, Qu R, Chai R, Shao R, Jin L, He L, Sun X, Wang L: MiRNA-320 in the human follicular fluid is associated with embryo quality in vivo and affects mouse embryonic development in vitro. Sci Rep 2015;5:8689.

-30 Hamam D, Ali D, Vishnubalaji R, Hamam R, Al-Nbaheen M, Chen L, Kassem M, Aldahmash A, Alajez NM: microRNA-320/RUNX2 axis regulates adipocytic differentiation of human mesenchymal (skeletal) stem cells. Cell Death Dis 2014;5:e1499.

-31 Schmitz ML, Kracht M: Cyclin-Dependent Kinases as Coregulators of Inflammatory Gene Expression. Trends Pharmacol Sci 2016;37:101-113.

-32 Hennessy EJ, Sheedy FJ, Santamaria D, Barbacid M, O’Neill LA: Toll-like receptor-4 (TLR4) downregulates microRNA-107, increasing macrophage adhesion via cyclin-dependent kinase 6. J Biol Chem 2011;286:25531-25539.

-33 Lu S, Wang MS, Chen PJ, Ren Q, Bai P: miRNA-186 inhibits prostate cancer cell proliferation and tumor growth by targeting YY1 and CDK6. Exp Ther Med 2017;13:3309-3314.

-34 Trohatou O, Zagoura D, Orfanos NK, Pappa KI, Marinos E, Anagnou NP, Roubelakis MG: miR-26a Mediates Adipogenesis of Amniotic Fluid Mesenchymal Stem/Stromal Cells via PTEN, Cyclin E1, and CDK6. Stem Cells Dev 2017;26:482-494.

-35 Trakala M, Arias CF, Garcia MI, Moreno-Ortiz MC, Tsilingiri K, Fernandez PJ, Mellado M, Diaz-Meco MT, Moscat J, Serrano M, Martinez AC, Balomenos D: Regulation of macrophage activation and septic shock susceptibility via p21(WAF1/CIP1). Eur J Immunol 2009;39:810-819.

-36 Mavers M, Cuda CM, Misharin AV, Gierut AK, Agrawal H, Weber E, Novack DV, Haines GK, 3rd, Balomenos D, Perlman H: Cyclin-dependent kinase inhibitor p21, via its C-terminal domain, is essential for resolution of murine inflammatory arthritis. Arthritis Rheum 2012;64:141-152.

-37 Handschick K, Beuerlein K, Jurida L, Bartkuhn M, Muller H, Soelch J, Weber A, Dittrich-Breiholz O, Schneider H, Scharfe M, Jarek M, Stellzig J, Schmitz ML, Kracht M: Cyclin-dependent kinase 6 is a chromatinbound cofactor for NF-kappaB-dependent gene expression. Mol Cell 2014;53:193-208.

-38 Torices S, Julia A, Munoz P, Varela I, Balsa A, Marsal S, Fernandez-Nebro A, Blanco F, Lopez-Hoyos M, Martinez-Taboada V, Fernandez-Luna JL: A functional variant of TLR10 modifies the activity of NFkB and may help predict a worse prognosis in patients with rheumatoid arthritis. Arthritis Res Ther 2016;18:221.

-39 Hoffmann A, Levchenko A, Scott ML, Baltimore D: The IkappaB-NF-kappaB signaling module: temporal control and selective gene activation. Science 2002;298:1241-1245.

-40 Whiteside ST, Epinat JC, Rice NR, Israel A: I kappa B epsilon, a novel member of the I kappa B family, controls RelA and cRel NF-kappa B activity. Embo J 1997;16:1413-1426. 